Meta

Journal des traducteurs

Translators' Journal

\title{
La traduction : classification et éléments d'analyse
}

\section{Amparo Hurtado Albir}

Volume 41, numéro 3, septembre 1996

URI : https://id.erudit.org/iderudit/001867ar

DOI : https://doi.org/10.7202/001867ar

Aller au sommaire du numéro

Éditeur(s)

Les Presses de l'Université de Montréal

ISSN

0026-0452 (imprimé)

1492-1421 (numérique)

Découvrir la revue

Citer cet article

Hurtado Albir, A. (1996). La traduction : classification et éléments d'analyse. Meta, 41(3), 366-377. https://doi.org/10.7202/001867ar

\section{Résumé de l'article}

Après un bref historique de la classification de la traduction, on expose les différents types de classification proposés par les théoriciens modernes. L'auteur présente ensuite sa propre classification qu'elle veut "intégratrice", c'est-à-dire que tous les éléments de catégorisation proposés s'interpénètrent pour caractériser et pour classer la traduction. d'utilisation que vous pouvez consulter en ligne.

https://apropos.erudit.org/fr/usagers/politique-dutilisation/ 


\title{
LA TRADUCTION: CLASSIFICATION ET ÉLÉMENTS D'ANALYSE
}

Amparo HurTado Albir

Université Autonome de Barcelone, Barcelone, Espagne

\begin{abstract}
Résumé
Après un bref historique de la classification de la traduction, on expose les différents types de classification proposés par les théoriciens modernes. L'auteur présente ensuite sa propre classification qu' elle veut «intégratrice», c'est-à-dire que tous les éléments de catégorisation proposés s'interpénètrent pour caractériser et pour classer la traduction.
\end{abstract}

Abstract

The author briefly retraces the history of translation typology, discusses typologies used by modern theoreticians then proposes her own "integrated" typology consisting of a combination of interconnected elements for classifying translations.

I. LA CLASSIFICATION DE LA TRADUCTION JUSQU'AUX PREMIÈRES THÉORIES MODERNES. CLASSIFICATIONS THÉMATIQUES ET CLASSIFICATIONS MÉTHODOLOGIQUES

La volonté de classifier la traduction n'est pas un fait récent et, tout au long de l'histoire, différentes propositions ont été faites: saint Jérôme, Vives, Fray Luis de León, Dryden, Schleiermacher ${ }^{1}$... On pourrait diviser les classifications proposées en deux grands blocs : classifications «thématiques» et classifications «méthodologiques».

Les classifications thématiques sont celles qui distinguent entre traduction religieuse et traduction profane, ou traduction scientifique et traduction littéraire...

Les classifications méthodologiques (par exemple, celle de Fray Luis de León; Vives; Dryden) font référence à la façon de traduire. C'est le type de classification qui prédomine tout au long de l'étape «préthéorique» (jusqu'à la deuxième moitié du $\mathrm{XX}^{\mathrm{e}}$ siècle). Les propositions peuvent être regroupées en trois catégories: 1) celles qui sont relatives à l'«opposition fondamentale» entre traduction littérale et traduction libre; 2) celles qui défendent ce que Steiner appelle la «iusta via media»; 3) celles qui préconisent la traduction du «sens» (même si pendant cette longue période on l'identifie avec le «contenu»).

Entre toutes, la classification la plus importante est celle qui est en rapport avec la polarisation entre la traduction littérale et la traduction libre. Il faut ajouter à cela que la réflexion porte essentiellement sur la traduction écrite, avec la prédominance, en plus, de la traduction littéraire.

\section{L'«ÈRE DE LA TRADUCTION» : LES NOUVELLES VARIÉTÉS DE TRADUCTION AU $\mathrm{XX}^{\mathrm{e}}$ SIÈCLE. CLASSIFICATIONS}

1. L'ESSOR DE LA TRADUCTION AU XX ${ }^{\mathrm{e}}$ SIÈCLE:

L'AVÉNEMENT DE NOUVELLES VARIÉTÉS DE TRADUCTION

Un grand changement se produit au $\mathrm{XX}^{\mathrm{e}}$ siècle avec l'essor de la traduction et l'avènement de nouvelles variétés de traduction: l'interprétation consécutive, l'interprétation 
simultanée, le doublage, la traduction automatique, le sous-titrage qui substitue les intertitres du cinéma muet...2

Par ailleurs, la traduction s'élargit à tous les domaines du savoir et on assiste à l'essor de la traduction dite spécialisée: la traduction scientifique, technique, juridique, économique, administrative...

\section{CLASSIFICATIONS PROPOSÉES}

À la suite de changements dans le monde de la traduction et de l'évolution parallèle de la réflexion théorique, les théories modernes abordent la classification de la traduction avec de nouvelles perspectives. Nous avons regroupé les classifications proposées en différents blocs selon l'élément considéré comme prioritaire (même s'il y a des classifications qui «s'entrecroisent») : 1) le changement de code; 2) le degré de traduisibilité; 3 ) les différences méthodologiques; 4) les différences de type textuel; 5) les différences de type textuel et méthodologique (qui conjuguent les deux); 6) celles qui prennent en compte différents éléments, et que l'on pourrait qualifier d'«intégratrices».

2.1. Classifications Par LE CHANGEMENT dE CODE

Nous considérons ici les classifications qui élargissent le terme «traduction» à n'importe quel processus de transformation entre codes distincts avec le maintien d'un invariant.

Jakobson (1959) distingue entre «traduction intersémiotique», traduction «intralinguistique» et traduction «interlinguistique». Ljudskanov (1969) conçoit la traduction comme un processus de transformation de signes et de maintien d'un invariant et il cherche des algorithmes polylingues valables tant pour la traduction humaine que pour la traduction mécanique. Le premier chapitre de After Babel de Steiner (1975), «Understanding as translation», abonde en exemples de «traduction intralinguistique»: par changements d'époque (la «traduction diachronique»), par changements de registre (les classes sociales, les enfants et les adultes, etc.), élargissant le concept de traduction à la communication en général, comme l'indique le titre du chapitre. Arcaini (1986) établit le rapport entre le signe linguistique et le signe iconique et il parle de la «lecture et de l'interprétation» (c'est-à-dire de la traduction) entre les codes verbaux et les codes iconographiques.

2.2. Classifications par le degré de traduisibilité

Rappelons que Neubert (1968) parle de traduction relative, partielle et optimale; House (1981) de «covert translation» («traduction camouflée»), celle qui jouit de la condition de texte original dans le milieu d'arrivée, puisque la fonction de l'original reste intacte, et «overt translation» («traduction patente»), qui n'a pas la condition de texte original et qui a besoin d'un deuxième niveau fonctionnel pour maintenir la même fonction.

2.3. CLASSIFICATIONS PAR DIFFÉRENCES MÉTHODOLOGIQUES

Ces classifications portent sur la méthode, c'est-à-dire sur la façon dont on traduit. Il convient de distinguer entre les propositions dichotomiques et les propositions plurielles.

Les propositions dichotomiques sont des classifications marquées par des pôles radicalement opposés :

- «Traduction littérale» contre «traduction libre», reprenant l'opposition traditionnelle ;

- «Traduction litterale» contre «traduction oblique», chez les tenants de la stylistique comparée (opposition faite sur le plan des langues);

- «Traduction sémantique» (vers l'auteur et propre aux textes expressifs) contre «traduction communicative» (vers le destinataire et propre aux textes informatifs et vocatifs) de Newmark;

- «Traduction camouflée» contre «traduction patente» de House; 
- «Traduction indirecte» (flexible et dépendante du contexte) contre «traduction directe» (notion fixe et indépendante du contexte) de Gutt (1991).

Les propositions plurielles sont celles qui établissent des classifications multiples:

- Catford (1965) propose différentes classifications: 1) Une distinction en fonction de l'extension de la traduction (traduction complète et traduction partielle). 2) Une distinction en fonction du niveau où se situe la traduction (traduction totale et traduction restreinte à un seul niveau: traduction phonologique, graphologique, grammaticale, lexicale). 3) Une distinction entre traduction mot à mot, traduction littérale et traduction libre (mais sur le plan des différences entre les langues). 4) Et une dernière distinction entre «traduction» (relative aux textes) et «transférence» (relative aux langues).

- Newmark (1988), même si chez lui l'essentiel est la différence entre traduction sémantique et traduction communicative, propose d'autres types de traduction tels que la traduction mot à mot, la traduction littérale, la traduction fidèle, la traduction-adaptation, la traduction libre, la traduction idiomatique 3 ...

- Hewson et Martin (1991) signalent toute une série d'«options ouvertes» : 1) la «réduction», lorsqu'un système culturel domine l'autre et que par conséquent on peut le reconvertir, contre la «marginalisation», lorsque le système culturel ne peut pas être reconverti; 2) l'«insertion», si l'on peut intégrer quelques valeurs d'un système dans l'autre système; 3) la «conversion», lorsqu'on peut employer des valeurs similaires.

2.4. ClassSIFICATIONS PAR DIFFÉRENCES DE TYPE TEXTUEL

Nous trouvons aussi dans ce bloc des propositions dichotomiques: Kade (1968), Koller (1979), Delisle (1980) distinguent entre la traduction des textes pragmatiques et la traduction des textes littéraires; Wilss (1977) différencie la traduction des textes dénotatifs et celle des textes connotatifs...

Mais il faut citer surtout la proposition tripartite de Reiss de 1971 et de 1976 . Reiss se base sur les fonctions de la langue de Bühler et elle propose une typologie tripartite et monofonctionnelle: textes au contenu prédominant (scientifiques, techniques), textes à fonction expressive prédominante (littéraires), textes à fonction conative prédominante (publicitaires); elle ajoute les textes «subsidiaires», qui ont un support non verbal (la traduction cinématographique, de l'opéra).

Il faut mentionner aussi la classification plurielle que propose Koller (1979) lorsqu'il affirme qu'il faut considérer plusieurs éléments de fonctionnement textuel: la fonction dominante de la langue, les caractéristiques du contenu, les caractéristiques linguistiques et stylistiques, les caractéristiques formelles et esthétiques, et les caractéristiques pragmatiques.

2.5. CLASSIFICATIONS PAR DIFFÉRENCES DE TYPE TEXTUEL ET DIFFÉRENCES MÉTHODOLOGIQUES

Nous introduisons ici les classifications qui assignent une méthode de traduction déterminée selon le type textuel dont il s'agit.

La classification la plus connue est sans doute celle de House, déjà citée. Rappelons que House propose une typologie textuelle duelle, textes idéationnels et textes interpersonnels (textes qui ne sont pas en rapport avec la culture de départ et textes en rapport avec la culture de départ), ainsi qu'une typologie méthodologique duelle («traduction camouflée» et «traduction patente»).

Newmark, en partant d'une classification tripartite des textes fondée sur les fonctions de la langue de Bühler (textes informatifs, expressifs, opératifs), y attribue des méthodes différentes (traduction sémantique et traduction communicative). 


\section{PROPOSITIONS «INTÉGRATRICES»}

Un autre aspect présente les classifications de la traduction que nous appelons «intégratrices», qui introduisent d'autres éléments dans la catégorisation, telle que la variété de «moyen ${ }^{4}$ ».

Holmes (1972) dans «The Name and Nature of Translation Studies ${ }^{\text {» }}$, même s'il s'agit d'un article axé sur l'analyse des études sur la traduction, propose aussi une classification de la traduction. Lorsqu'il parle des études théoriques partielles, il distingue six variables parmi lesquelles la variable de «moyen» (traduction mécanique, traduction humaine, traduction orale, traduction écrite) et la variable de «type» (traduction littéraire, de textes théologiques, scientifiques ${ }^{6}$ ). Holmes n'entre pas dans le détail de la classification, mais il introduit la variable de «moyen» qui est, à notre avis, essentielle.

House, lorsqu'elle introduit les huit dimensions pour définir la fonction textuelle (fondées sur Crystal et Davy) propose aussi le «moyen» comme une de ces dimensions et elle distingue (en reprenant la proposition de Gregory) entre «moyen simple» et «moyen complexe».

Snell-Hornby (1988) se prononce clairement pour une conception intégratrice des études sur la traduction, et elle propose une classification intégratrice de tout le champ de la traduction?.

Une autre proposition qui prétend reprendre toutes les manifestations de la traduction est celle de Rabadán (1991). Rabadán insiste sur les variétés par le changement de «moyen» qui produisent des «modes» différents (dans la ligne de Gregory et Carroll; Halliday; Crystal et Davy). La division de base est établie entre trois classes de «moyens» (le son, la graphie et l'image) qui sont les «modes primaires» (textes oraux immédiats, textes écrits et textes iconiques), ce qui donne dans la traduction: l'interprétation, la traduction écrite, la traduction d'affiches publicitaires et la traduction cinématographique. Elle reprend aussi la distinction de Crystal et Davy de «mode complexe» (lorsque le texte participe de plusieurs «modes») : les conférences, les textes dramatiques pour la scène, les mots croisés, les textes cinématographiques... En suivant la proposition de Titford (1982) et de Mayoral (1986), elle parle de «traduction subordonnée» : les variétés de traduction où interviennent d'autres codes outre le linguistique (l'iconique, le musical) et qui conditionnent l'équivalence de traduction.

\section{III. ÉLÉMENTS DE CATÉGORISATION. LIMBRICATION DES CATÉGORIES DE CLASSIFICATION}

\section{POUR UNE CLASSIFICATION INTÉGRATRICE DE TOUTES LES VARIÉTÉS DE TRADUCTION}

Notre proposition de classification se place dans cette ligne intégratrice de toutes les variétés de traduction, introduisant la variété de «mode». La question est beaucoup plus complexe que ce que l'on pourrait d'abord penser, puisqu'il y a des catégories qui s'interpénètrent dans la classification. Il est évident que, du point de vue théorique et méthodologique, les catégories du type «traduction technique» sont insuffisantes. Considérons, par exemple, la traduction d'un texte d'informatique. Il nous intéressera de connaître :

Le fonctionnement textuel de l'original, c'est-à-dire s'il s'agit d'un article d'une revue spécialisée, d'un article de vulgarisation, d'un mode d'emploi, d'une publicité adressée aux spécialistes ou à des usagers non spécialistes (et en plus si c'est une affiche publicitaire ou un spot), d'une conférence pour un congrès, d'un documentaire pour la télévision... C'est-à-dire qu'outre la variable de la fonction textuelle (en rapport avec les fonctions du langage), il faut prendre en compte la variable de «mode» de l'original. 
Le «mode» dans lequel s'effectue la traduction du texte en question: si c'est par écrit, ou oralement (et de quel genre: traduction à vue, ou simultanée, consécutive); ou bien, si l'original est audiovisuel, s'il s'agit d'un doublage, sous-titrage, ou d'une interprétation simultanée cinématographique. Le même texte original peut donc être traduit avec des «modes» différents. Par conséquent, au «mode» du texte original, il faut ajouter ce que nous appelons le «mode traducteur».

Mais pour avoir une vision complète de la traduction d'un texte, il faut également considérer la finalité de la traduction: traduit-on le texte pour une commande professionnelle ou s'agit-il d'une utilisation pédagogique? D'autre part, du point de vue de l'individu qui a fait la traduction, on se demandera s'il a traduit vers sa langue maternelle ou vers une langue étrangère. Et enfin, on s'interrogera aussi sur la façon dont le texte a été traduit, c'est-à-dire sur la méthode suivie par le traducteur.

Les éléments à considérer dans la catégorisation de la traduction humaine interlinguistique ${ }^{8}$ sont donc les suivants :

1) Le fonctionnement textuel de l'original (le type de texte, le mode, le registre, le champ thématique, etc.).

2) Le «mode de la traduction», qui coïncide parfois avec celui de l'original mais pas toujours. Ce premier élément nous fait proposer une distinction essentielle, les «modalités de traduction», différentes quant au «mode» de la traduction: la traduction écrite, la traduction à vue, l'interprétation simultanée, l'interprétation de liaison, le doublage, le sous-titrage, la traduction musicale, etc.

3) La nature de la fonction de la traduction (est-elle ou non une fin en soi ?).

4) La direction du processus traducteur (vers la langue maternelle ou vers la langue étrangère).

5) La méthode employée pour traduire le texte original.

De toutes ces variables, le mode traducteur, la nature de la fonction de la traduction, la direction du processus de la traduction, et la méthode employée sont les variables propres qu'introduit la traduction par rapport au texte original.

Il faut considérer l'imbrication qui se produit entre toutes ces catégories: toutes s'entremêlent pour configurer toute opération traduisante. Cette considération est fondamentale pour savoir quelles sont les caractéristiques de la traduction que l'on veut analyser ou que l'on veut produire.

\section{VARIÉTÉS SELON LA NATURE DE LA FONCTION DE LA TRADUCTION}

On peut d'emblée faire la distinction entre la fonction communicative professionnelle de la traduction, où traduire est une fin en soi, et la traduction dans sa fonction utilitaire, où la traduction remplit une fonction médiatrice pour une autre fin. On peut donc distinguer:

1) La traduction professionnelle, où traduire est un but en soi, sans oublier qu'elle peut remplir différentes fonctions selon le but poursuivi ou le public visé ( $c f$. infra VII.5.).

2) La traduction «utilitaire», dans ses différents aspects: a) L'enseignement de la traduction professionnelle, où le but est d'apprendre à traduire; b) la «traduction pédagogique», c'est-à-dire l'emploi de la traduction de textes en didactique des langues, où le but est un perfectionnement linguistique; c) la «traduction intériorisée», la comparaison (spontanée et inconsciente) avec la langue maternelle lorsqu'on apprend une langue étrangère (surtout au début de l'apprentissage); d) la «traduction explicative», l'usage ponctuel, délibéré et conscient de la traduction comme mécanisme d'accès à la signification d'un mot ou d'une expression d'une autre langue. 
3) La «traduction naturelle», c'est-à-dire l'habilité innée et rudimentaire de médiation entre langues que possèdent les parlants plurilingues ( $c f$. Harris et Sherwood, 1978).

Dans toutes ces variétés, ce qui change est la fonction (utilitaire ou non) mais aussi la nature du processus cognitif (spontané, délibéré), ainsi que la fonction dans les processus d'apprentissage.

\section{VARIÉTÉS SELON LA DIRECTION DU PROCESSUS DE LA TRADUCTION}

Il s'agit à présent d'une distinction en rapport avec l'individu qui traduit: s'il traduit vers sa langue maternelle (la «version», considérée comme la vraie traduction) ou vers une langue qui n'est pas sa langue maternelle (le «thème»).

En ce qui concerne le «thème», il convient de distinguer entre son utilisation professionnelle et son emploi didactique. Dans son emploi didactique, il faut encore distinguer entre son emploi didactique des langues (où il jouit d'une très longue tradition en tant qu'exercice de perfectionnement linguistique) et son emploi dans la formation de traducteurs, où il est aussi en rapport avec son rôle dans la vie professionnelle? ${ }^{9}$.

De toutes façons, il faut nuancer le degré de bilinguisme de l'individu (il existe des cas où le traducteur est capable de traduire dans les deux sens), la modalité de traduction dont il s'agit (en interprétation, on travaille plus dans les deux sens), ainsi que la combinaison linguistique en question (dans le cas de combinaisons peu courantes, le traducteur est parfois obligé de traduire dans les deux sens).

\section{VARIÉTÉS SELON LE TYPE TEXTUEL. LES «PROTOTYPES» DE TRADUCTION. LES CATÉGORIES DYNAMIQUES D'ANALYSE}

La variable de type textuel configure ce que j'appelle les «types de traduction», ou, mieux, les «prototypes de traduction».

\section{CARACTÉRISATION DU DÉBAT}

La question des typologies textuelles concernant la traduction a été largement débattue 10; la question est très complexe et elle mérite un traitement détaillé er exhaustif. Nous allons nous limiter à signaler l'état actuel du débat. Trois points essentiels, à notre avis, caractérisent la question des types textuels en traduction. En premier lieu, contre la rigidité classificatrice en types monofonctionnels, il faut considérer la «multifonctionnalité» des textes, proposée par des auteurs comme Reiss (1981), Hatim et Mason (1990) et Rabadán (1991); dans cette multifonctionnalité, il existe une hiérarchie de fonctions, où il faudra chercher la (les) fonction(s) prioritaire(s) dans chaque cas. En deuxième lieu, la revendication de «prototypes textuels», plutôt que de «types» tels que proposé par Neubert (1985), Snell-Hornby (1988). En troisième lieu, la nécessité d'établir des catégories d'analyse (plutôt que des classifications rigides et statiques), considérées, en plus, comme des catégories dynamiques (à employer selon les cas); des catégories qui puissent être employées pour tracer, comme disait House, le «profil textuel» de l'original.

\section{LA CONSTRUCTION DU SENS CONTEXTUEL. CATÉGORIES D'ANALYSE}

Le tableau 1 explique la progression de la construction du sens contextuel (à partir des significations hors contexte), avec la participation imbriquée des dimensions intratextuelles et extratextuelles (ou contextuelles). 


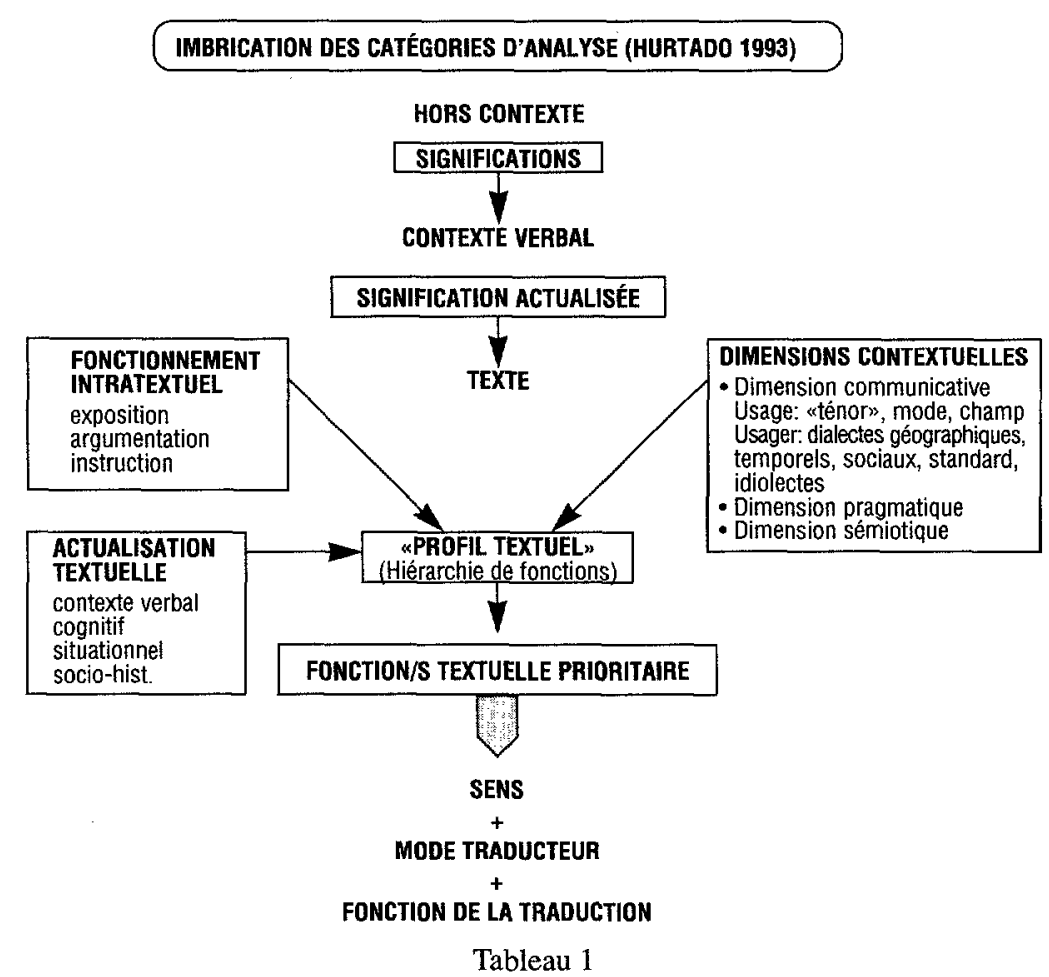

En ce qui concerne les dimensions intratextuelles, il convient d'analyser l'implication des catégories d'exposition, argumentation, et instruction. En plus, il faut tenir compte des mécanismes d'actualisation textuelle, qui conjuguent l'intervention des contextes: le contexte verbal (ou cotexte), le contexte cognitif, le contexte situationnel, et le contexte général socio-historique. Quant aux dimensions contextuelles (qui explicitent les conséquences du contexte situationnel et socio-historique), nous reprenons les catégories de Hatim et Mason (1990) : 1) la dimension communicative, avec les variables d'usage («ténor», champ, mode) et d'usager (les dialectes géographiques, temporels, sociaux, standard et l'idiolecte); 2) la dimension pragmatique, concernant les actes de parole; 3) la dimension sémiotique, concernant l'intertextualité, intégrant les variables de «texte», «discours» et «genre»11.

L'imbrication de ces éléments d'analyse, dont la synthèse est le sens, trace le profil textuel de l'original. À cela, il faudra ajouter le mode traducteur et la fonction de la traduction.

Le fait de proposer ces catégories dynamiques, critiquant les compartimentations rigides, n'est pas contradictoire avec la distinction que l'on fait dans le monde professionnel entre traduction de textes spécialisés (techniques, scientifiques, économiques, etc.), traduction de textes «généraux», et traduction de textes littéraires ${ }^{12}$, puisque la traduction de ces textes est en rapport avec la nécessité de compléments cognitifs différents, exigeant du traducteur des compétences différentes, et par conséquent un «profil» différent ${ }^{13}$. 


\section{VARIÉTÉS SELON LA MÉTHODE EMPLOYÉE}

Tout comme la question des typologies textuelles en traduction, la question de la méthode de traduction est complexe et tout à fait digne d'un traitement à part; nous nous limiterons ici à établir ce qui, à notre avis, sont les prémisses fondamentales du débat autour de ce sujet.

1) La nécessité d'annuler les dichotomies méthodologiques du genre traduction littérale contre traduction libre, traduction sémantique contre traduction communicative, traduction camouflée contre traduction patente. À nos yeux, ce sont des dichotomies en rapport avec toute une série d'oppositions, considérées à l'heure actuelle comme non pertinentes, telles que l'opposition entre fond et forme, entre langue et parole, entre émetteur et récepteur.

2) La critique de la compartimentation méthodologique selon le type de texte, c'està-dire la croyance selon laquelle les textes techniques ou scientifiques, par exemple, exigent une méthode littérale, et la poésie, l'interprétation simultanée, la traduction à vue, une méthode libre. En outre, ces types de considérations sont basés sur des typologies textuelles extrêmement rigides; d'ailleurs, elles ne tiennent compte que du résultat de la traduction et non du processus suivi. Il faudrait plutôt parler de conditionnements spécifiques (et non de méthodes différentes).

3) Le besoin d'établir la distinction entre les différences de résultat (la traduction dans sa comparaison avec le texte original) et les différences de processus. Ce qui compte, du point de vue de l'analyse de la méthode de traduction, ce sont les changements dans le processus (évidemment analysables à partir des résultats); il faut donc défendre la nécessité de l'étiologie dans l'analyse.

4) La relation existante entre le processus traducteur dévéloppé et la méthode de traduction employée, et dans ce sens la prise en compte du fait que les changements de méthode supposent des changements dans le déroulement du processus traducteur. La définition, donc, de la méthode traductive comme le déroulement d'un processus traducteur determiné régulé par un principe.

5) La classification des méthodes par rapport à la considération d'objectifs différents, et non comme des formes opposées, alternatives et irréconciliables de traduire. Ainsi, reprenant les dénominations traditionnelles, nous proposons la distinction entre méthode littérale et méthode libre, différentes de la méthode interprétative-communicative (où le traducteur est censé comprendre le sens de l'original et le réexprimer de façon à produire le même effet chez le destinataire de la traduction ${ }^{14}$ ). La méthode littérale, le simple décodage linguistique de l'original. La méthode libre (avec ses variantes fondamentales de traduction-adaptation et version libre) lorsqu'on veut faire un changement d'époque, de milieu socio-culturel, de genre, de registre... qui parcourt tout le texte ${ }^{15}$.

Chacune de ces méthodes poursuit des objectifs différents. Il existe un lien entre la fonction de la traduction (qui peut être la même ou non que celle de l'original) et la méthode employée. Un changement de fonction de la traduction peut amener le traducteur à employer une méthode littérale ou libre, qui serait pertinente par rapport à la fonction de la traduction 16 .

6) La distinction entre «méthode», «stratégie» et «technique de traduction». Nous croyons que la «méthode» a un caractère supra-individuel (et parfois inconscient). La «stratégie de traduction» possède un caractère individuel (conscient ou inconscient) puisque c'est le mécanisme concret utilisé pour résoudre un problème de traduction: reformuler à haute voix, répéter la même idée, imaginer la situation de communication, utiliser une source déterminée de documentation... La «technique de traduction» est l'application concrète, visible dans le résultat: explicitation, adaptation ponctuelle, omission, etc. 
VIII. VARIÉTÉS SELON LE MODE TRADUCTEUR. MODÈLE CLASSIFICATEUR DE LA TRADUCTION CONSIDÉRANT LE MODE TRADUCTEUR: LES MODALITÉS DE TRADUCTION

Nous proposons un modèle classificateur de la traduction considérant la dernière variable, le «mode traducteur» qui génère ce que nous appelons les modalités de traduction.

Nous considérons le «mode traducteur» non comme celui de l'original, mais comme celui qui introduit le processus traducteur. Le mode traducteur peut être: 1) simple, si on maintient le même que l'original; 2) complexe, s'il y a un changement par rapport à l'original; 3) subordonné, lorsqu'il se produit, déjà dans l'original, un mélange de moyens (ce qui produit toute une série de conditionnements dans la traduction; d'où le terme «subordonné»); le mode traducteur subordonné peut être à son tour simple (s'il maintient celui de l'original) ou complexe (s'il introduit des variations).

Les tableaux présentés à la fin de cet article proposent une classification des modalités de traduction, signalant leurs particularités en fonction de toute une série de paramètres: 1) le «moyen» de l'original; 2) le mode de l'original; 3) le mode traducteur (le paramètre déterminant); 4) la situation d'emploi; 5) les compétences communicatives requises du traducteur; 6) les conditionnements spécifiques.

Ces tableaux mettent en relief quelques modalités qui ont été considérées traditionnellement comme ayant un mode simple, mais qui ont en réalité un mode complexe. La classification reste donc:

1) Mode traducteur simple : la traduction écrite, l'interprétation simultanée, l'interprétation de liaison, le «chuchotage».

2) Mode traducteur complexe: la traduction à vue, l'interprétation consécutive.

3) Mode traducteur subordonné simple : la traduction théâtrale pour la scène, le doublage, la traduction de BD, d'affiches publicitaires et de mots croisés.

4) Mode traducteur subordonné complexe: le sous-titrage et le «surtitrage» (pour l'opéra).

Il peut se produire évidemment des «croisements» de ces modalités. Par exemple, l'interprète en simultané en vient à effectuer de la traduction à vue si l'orateur lit son discours ou sa conférence et l'interprète, possédant l'original écrit, le lit et le traduit à haute voix; ou lorsque dans la traduction de films (et aussi pour des pièces de théâtre), on effectue l'interprétation simultanée (spontanée ou préparée); ou bien le sous-titrage de chansons dans les films...

Quelquefois, les «croisements» se produisent par des changements de la fonction de la traduction. C'est le cas, par exemple, de la traduction théâtrale devant être lue (où le client est une maison d'édition et le destinataire un public lecteur) produisant, par exemple, une traduction «philologique»; ou de la traduction de «scénarios» ou de chansons destinés à être lus (par exemple, ou publiés par une maison d'édition)...

Une autre variation est constituée par les «intertitres traduits» qui s'effectuaient avec le cinéma muet, ou ceux qui se faisaient aux États-Unis aux débuts du cinéma sonore avec les films étrangers (comme une consécutive toutes les 15 ou $20 \mathrm{~min}$ ).

Il faut encore insister sur le fait que tous les éléments de catégorisation proposés (les prototypes, le mode traducteur, la nature de la fonction de la traduction, etc.) s'interpénètrent pour caractériser et pour classer la traduction. Prenons, par exemple, la traduction d'un texte «littéraire» comme Cyrano de Bergerac; les solutions de traduction seront différentes s'il s'agit de le traduire avec la modalité de traduction écrite, ou de doublage, ou de sous-titrage... À son tour, la traduction cinématographique n'est pas une unité statique, car on traduit des classiques de la littérature, des feuilletons télévisés, des spots publicitaires, des dessins animés, des documentaires... Il faut insister aussi sur le fait que 
le mode traducteur, et la classification en modalités qui en découle, est à notre avis une catégorie dominante pour traiter et pour classer la traduction.

\begin{tabular}{|c|c|c|c|c|c|c|}
\hline MODALITÉ & $\begin{array}{c}\text { MOYEN } \\
\text { (DE L'ORIGINAL) }\end{array}$ & $\begin{array}{c}\text { MODE } \\
\text { (DE L'ORIGINAL) }\end{array}$ & MODE TRADUCTEUR & SITUATTON D'USAGE & $\begin{array}{c}\text { COMPÉTENCES } \\
\text { COMMLNICATIVES }\end{array}$ & CONDITIONNEMENTS \\
\hline $\begin{array}{l}\text { traduction } \\
\text { écrite }\end{array}$ & Écrit & $\begin{array}{l}\text { simpie (et complexe) } \\
\text { Écrit pour être lus } \\
\text { (et/ou «parlés) }\end{array}$ & $\underset{\text { secritécrit }}{\text { simple }}$ & $\begin{array}{l}\text { - fous les cas de } \\
\text { communication écrite } \\
\text { (pour être lue } \\
\text { et/ou upartéen) } \\
\text { - tous les types textuels }\end{array}$ & $\begin{array}{l}\text { comprétension écrite } \\
\text { expression écrite }\end{array}$ & $\begin{array}{c}\text { ceux de ia } \\
\text { communication } \\
\text { écrite (code écrit, } \\
\text { «rieillissement», etc.) }\end{array}$ \\
\hline traduction à yue & Errit & $\begin{array}{l}\text { simple et complexe } \\
\text { Écrit pour être lut } \\
\text { et/oı «pariés }\end{array}$ & $\begin{array}{l}\text { complexe } \\
\text { écrit-oral }\end{array}$ & 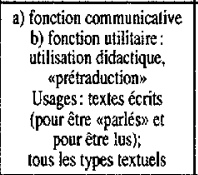 & $\begin{array}{l}\text { compréhension écrite } \\
\text {-expression orale }\end{array}$ & $\begin{array}{c}\text { Dans sa fonction } \\
\text { comunicative: ceux de } \\
\text { l'expression orale } \\
\text { (conte oral. immé́diatement, } \\
\text { etc.) }\end{array}$ \\
\hline interprétalion simultanée & Oral & $\begin{array}{l}\text { simple (et complexe) } \\
\text { Oral spontané } \\
\text { (et non spontané) }\end{array}$ & $\begin{array}{c}\text { simple } \\
\text { oral-oral }\end{array}$ & $\begin{array}{l}\text { - conférences et discours } \\
\text { - débats ayec quelques } \\
\text { ou plusieurs interlicuteurs }\end{array}$ & $\begin{array}{l}\text { comprétension orale } \\
\text {-expression orale }\end{array}$ & $\begin{array}{l}\text { - ceux de la communication } \\
\text { orale (compréhension } \\
\text { el expression) : } \\
\text { non témanence, rapidité } \\
\text { + la «simultaneites }\end{array}$ \\
\hline interprétation consécutive & Otal & $\begin{array}{l}\text { simple et complexe } \\
\text { Oral spontané et } \\
\text { non spontané }\end{array}$ & $\begin{array}{c}\text { complexe } \\
\text { oralécril } \\
\text { (prise de notes)-oral }\end{array}$ & $\begin{array}{l}\text { - conférences, discours } \\
\text { - débats avec peur } \\
\text { d'interlocuteurs }\end{array}$ & $\begin{array}{l}\text { compréhensicn orale-- } \\
\text { synthesse graphique } \\
\text {-expression orale }\end{array}$ & $\begin{array}{c}\text { - ceux de la compréhension } \\
\text { orale } \\
\text { - synthèse des notes } \\
\text { - la cpostériorités }\end{array}$ \\
\hline interpretation de liaison & Oral & $\begin{array}{c}\text { simple } \\
\text { Oral spontané }\end{array}$ & $\begin{array}{l}\text { simple } \\
\text { oral-oral }\end{array}$ & $\begin{array}{c}\text { conversations } \\
\text { (politiques, d'affaires, etc.) }\end{array}$ & $\begin{array}{l}\text { compréhension orale } \\
\text {-expression orale }\end{array}$ & $\begin{array}{c}\text { - ceux de la conmmunication } \\
\text { orale } \\
\text { - les mécanismes } \\
\text { conversationnels de chaque } \\
\text { langue (sauf daus la } \\
\text { variété de } 35 \text { s style indirect) }\end{array}$ \\
\hline "wchuchotage" & Oral & $\begin{array}{l}\text { simple et complexe } \\
\text { Oral spontané } \\
\text { et non spontané }\end{array}$ & $\begin{array}{l}\text { simple } \\
\text { oral-oral }\end{array}$ & $\begin{array}{l}\text { - conférences, discours } \\
\text { - débats ayec plusieurs ou } \\
\text { peu d'interlocitteurs }\end{array}$ & $\begin{array}{c}\text { conpréhension orale } \\
\rightarrow \text {-xpression orale }\end{array}$ & $\begin{array}{l}\text { - ceux cie la communication } \\
\text { orale } \\
\text { - fragmentation el voix basse } \\
\text { (synthèse) }\end{array}$ \\
\hline $\begin{array}{l}\text { traduction théatrale } \\
\text { pour la mise en scène }\end{array}$ & Ecrit (oral) & $\begin{array}{c}\text { complexe } \\
\text { Écrit pour être joúé }\end{array}$ & $\begin{array}{c}\text { simple subordonné } \\
\text { ecrit pour ête joué-ecrit } \\
\text { pour être jové }\end{array}$ & représentation scénique & $\begin{array}{c}\text { compréhension } \\
\text { dramaturgique } \\
\text {-expression dramaturgique }\end{array}$ & $\begin{array}{c}\text { conditionnements } \\
\text { dramaturgiques : éléments } \\
\text { prosodiques, réception } \\
\text { immódiate, elc. }\end{array}$ \\
\hline doublage & Audiovisuel & $\begin{array}{l}\text { complexc } \\
\text { - Oral } \\
\text { - Yisuel }\end{array}$ & $\begin{array}{l}\text { simple subordonné } \\
\text { audiovisuel-oral } \\
\text { subordonné } \\
\text { (audiovisuel) }\end{array}$ & adaptation & $\begin{array}{l}\text { conppréhension orale } \\
\text { (audiovisuetle) } \\
\text {-expression orale } \\
\text { (audiovisuelle) }\end{array}$ & $\begin{array}{l}\text { - synchronie et adaptation } \\
\text { aux mouvements articulatoires } \\
\text { - différences de etempo* } \\
\text { de chaque langue }\end{array}$ \\
\hline soustilrage & Audiovisuel & $\begin{array}{l}\text { complexe } \\
\text { - Oral } \\
\text { - Visuel }\end{array}$ & $\begin{array}{l}\text { complexe subordonné } \\
\text { audiovisueléćrit } \\
\text { subordonné }\end{array}$ & synclaronisation & $\begin{array}{l}\text { compréhension orale } \\
\text { (audiovisuelle) } \\
\text {-expression écrite } \\
\text { (audiovisueile) }\end{array}$ & $\begin{array}{l}\text { - synchronie avec la } \\
\text { bande sonore } \\
\text { - limilation du nombre } \\
\text { de caractères graphiques } \\
\text { - limitation du changement } \\
\text { de code (oral-écrit) }\end{array}$ \\
\hline $\begin{array}{c}\text { traduction de } \\
\text { bandes-dessinúe }\end{array}$ & iconique-graphique & $\begin{array}{l}\text { complexe } \\
\text { - Image } \\
\text { - Ecrit }\end{array}$ & $\begin{array}{l}\text { simple subordonné } \\
\text { écrit/inage- } \\
\text { érit subordonté } \\
\text { à l'image }\end{array}$ & $\begin{array}{l}\text { traduclion de BD } \\
\text { pour sa diffusion }\end{array}$ & 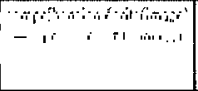 & 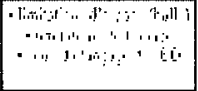 \\
\hline $\begin{array}{l}\text { traduction d'affiches } \\
\text { publicitaires }\end{array}$ & iconig̨ue-graphique & $\begin{array}{l}\text { complexe } \\
\text { - Image } \\
\text { - Écrit }\end{array}$ & $\begin{array}{l}\text { simple subordonné } \\
\text { écrit/image-_crit } \\
\text { subordonné à l'image }\end{array}$ & \begin{tabular}{|c|} 
Iraduction d'affiches \\
publicitaires pour leur diffusion
\end{tabular} & $\begin{array}{l}\text { compréhension écrite } \\
\text { (image)-expression écrite } \\
\text { (image) }\end{array}$ & $\begin{array}{l}\text { - symbiose entre image et } \\
\text { message linguistique } \\
\text { - ceux des messages } \\
\text { publicitaires (créativité, } \\
\text { jeux de mols) }\end{array}$ \\
\hline $\begin{array}{l}\text { traduction de rébus, } \\
\text { mots croisés }\end{array}$ & $\begin{array}{l}\text { iconique- } \\
\text { graphique }\end{array}$ & $\begin{array}{c}\text { complexe } \\
\text { Inage + Ecrit }\end{array}$ & $\begin{array}{l}\text { simple subordonné } \\
\text { image + écrit-image } \\
\text { +écrit }\end{array}$ & $\begin{array}{c}\text { · isolémentent (rare) } \\
\text { - inséré dans un autre texte }\end{array}$ & $\begin{array}{l}\text { corpprétension visuelle } \\
\text { (lecture)-expression } \\
\text { visuelle (êcrite) }\end{array}$ & $\begin{array}{c}\text { subordination de } \\
\text { l'écriture à limage }\end{array}$ \\
\hline Iraduction musicale & $\begin{array}{l}\text { - Musique } \\
\text {-Oral }\end{array}$ & $\begin{array}{l}\text { complexe } \\
\text { - Musique } \\
\text { - Oral }\end{array}$ & $\begin{array}{l}\text { simple subordoriné } \\
\text { oral/musique- } \\
\text { oral/musique }\end{array}$ & pour être chantée & $\begin{array}{c}\text { compréhension orale/musicale } \\
\text {-expression } \\
\text { orale /musicale }\end{array}$ & mesures, tons, etc. \\
\hline ksur-titragen musical & - Musique & $\begin{array}{l}\text { complexe } \\
\text { - Musique } \\
\text { - Oral }\end{array}$ & $\begin{array}{l}\text { complexe subordonné } \\
\text { oral/musique-écrit }\end{array}$ & $\begin{array}{c}\text { pour être lu } \\
\text { synchroniquement } \\
\text { à Ja chanson }\end{array}$ & $\begin{array}{l}\text { compréhension } \\
\text { orale/musicale-- } \\
\text { expression érite }\end{array}$ & $\begin{array}{l}\text { - synchronie avec la chanson } \\
\text { - limitation du nombre } \\
\text { de caracteres graphiques }\end{array}$ \\
\hline
\end{tabular}

\section{Notes}

1. Rappelons que saint Jérôme faisait une distinction entre «traduction profane» et «traduction religieuse»; distinction qui subsistera pendant tout le Moyen Âge (et même à la Renaissance) en considérant qu'il s'agit de «traductions» différentes. Vives différenciait entre les versions qui ne tiennent compte que du «sens», d'autres qui considèrent la «phrase» et la «diction», et un troisième genre d'équilibre entre la «substance» et les mots, lorsque, d'après lui, les mots ajoutent «force» et «grâce» au sens. Fray Luis de León distinguait 
entre «trasladar» et «declarar» : «trasladar» où, d'après lui, il faut être «fidèle» et «exact» et «si cela est possible compter les mots pour en rendre autant d'autres»; «declarar», c'est-à-dire «jouer avec les mots, ajoutant et enlevant à notre volonté». Dryden propose la distinction entre «métaphrase» (traduction mot à mot), «paraphrase» (traduction du «sens») et «imitation» («liberté» de varier la forme et le «sens»). Schleiermacher distingue entre la traduction de textes commerciaux, littéraires et scientifiques...

2. L'interprétation consécutive a été utilisée pour la première fois pendant la Conférence de paix de 1919; l'interprétation simultanée a été employée par la première fois dans la Conférence Internationale du travail de 1927 et dans la Conférence Mondiale de l'énergie de 1930, mais instaurée définitivement au cours du procès de Nuremberg; le doublage a été commencé en 1929; la traduction automatique en $1954 .$. .

3. En plus, Newmark introduit: le «service translation» (le «thème»), la traduction de poésie en prose, la traduction-information, la traduction cognitive, la traduction académique.

4. Reiss 1'avait déjà introduite en $1971:$ les textes «subsidiaires»

5. «The Name and Nature of Translation Studies» a été présenté pour la première fois au cours du Third International Congress of Applied Linguistics, qui a eu lieu à Copenhague en 1972. Le texte a été publié à maintes reprises $(1972,1975,1987)$; je cite l'édition recueillie dans Translated! Papers on Literary Translation and Translation Studies, Rodopi, Amsterdam, 1988, qui reprend la version de 1975.

6. Les quatre autres variables sont: langue et culture, niveau, problème, époque.

7. Rappelons que, à l'horizontale, elle situe un vaste spectre de textes, sans divisions, avec une transition graduelle; à la verticale, elle propose un modèle stratifié, de macro-niveaux à micro-niveaux, qui va du plus général (niveau A) au plus spécifique (niveau D). En ce qui concerne le niveau A, celui des «aires conventionnelles» (traduction littéraire, traduction générale, traduction des langages de spécialité), Snell-Hornby critique le fait que, traditionnellement, on les a considérées trop séparément: elle signale, à juste titre, que la traduction littéraire et la traduction spécialisée ont été considérées comme des domaines opposés et que cela n'est pas vrai puisqu'il y a des phénomènes, comme par exemple l'allitération, qui existent dans tous les textes. Dans le niveau B, celui de la prototypologie des textes de base, elle va de la Bible à la technologie moderne, en passant par la traduction cinématographique; c'est peut-être ici que la division et la situation «boitent», bien qu'elle insiste sur la transition graduelle. Elle signale, en plus, qu'il existe des variétés qu'elle ne présente pas, tout en reconnaissant qu'il s'agit d'un essai pour aborder tout le domaine de la traduction.

8. Je laisse donc de côté les variétés de traduction automatique, traduction intersémiotique et traduction intralinguistique.

9. Le marché du thème est beaucoup plus réduit pour le traducteur que celui de la version, mais il existe: la traduction commerciale, la traduction jurée... C'est pourquoi on l'introduit dans la formation des traducteurs, comme exercice de perfectionnement linguistique mais aussi par nécessité professionnelle, en rapport avec le marché du travail.

10. La pionnière est K. Reiss, représentante claire d'une approche typologique en théorie de la traduction.

11. Rappelons que, d'après Hatim et Mason, le «texte» est la «série de fonctions communicatives structurées qui forment un tout»; le discours étant les «façons de parler ou d'écrire adoptant une attitude particulière en fonction de l'aire socioculturelle (par exemple, le discours raciste, le discours officiel); et le «genre», les «formes conventionnelles de textes associées à des types particuliers d'événements sociaux» (par exemple, le sonnet, les recettes de cuisine, le discours politique).

12. Ce que Snell-Hornby appelle les «aires conventionnelles».

13. Pensons, par exemple, que les textes techniques posent des problèmes concrets par la complexité informative et l'emploi d'éléments terminologiques, ce qui exige du traducteur des connaissances extralinguistiques et linguistiques déterminées.

14. Voir sur ce sujet A. Hurtado Albir «iLibertad o servidumbre? Traducir el sentido, ésa es la cuestión», in M. Lederer, F. Israël (dir.) : La liberté en traduction, Paris, Didier Érudition, Coll. Traductologie 7, 1991, 263-280.

15. Il est important de distinguer la traduction-adaptation, résultat d'une méthode libre délibérée, justifiée et qui parcourt tout le texte, des adaptations ponctuelles (linguistiques ou extralinguistiques) pour produire le même effet chez le destinataire, résultat d'une méthode interprétative-communicative. Il faut signaler, en plus, qu'il existe des cas où la seule solution communicative, pour maintenir la même fonction de l'original et produire le même effet chez le destinataire, est la traduction-adaptation; c'est le cas des textes où tout tourne autour du référent socioculturel (par exemple, une pièce de théâtre basée sur l'actualité sociopolitique d'un pays).

16. Par exemple, la traduction d'un texte publicitaire pour faire la publicité du même produit dans un autre pays (même fonction) demanderait l'emploi d'une méthode interprétative-communicative: bien comprendre l'original et le reformuler, en faisant toutes les adaptations linguistiques, et même iconiques, nécessaires pour garder la même fonction et produire le même effet chez le destinataire de la traduction; or, si la finalité était tout simplement de faire comprendre à un client ou à un ami, ce que «dit» ce texte publicitaire (changement de fonction), il serait licite d'employer une méthode littérale. Le même cas se produirait avec la traduction d'un 
contrat pour son utilisation dans une entreprise (même fonction) où l'on suivrait les normes et les formules propres aux contrats dans le milieu d'arrivée, ou s'il s'agissait d'une traduction pour l'utiliser dans un procès (changement de fonction) où les juges demandent parfois une traduction plus littérale pour pouvoir suivre point par point le document original.

\section{RÉFÉRENCES}

ARCAINI, E. (1986) : Analisi linguistica e traduzione, Bologna, Patron.

CATFORD, J. C. (1965) : A Linguistic Theory of Translation, Oxford University Press.

DELISLE, J. (1980): L'analyse du discours comme méthode de traduction, Éditions de l'Université d'Ottawa.

GUTT, E. A. (1991) : Translation and Relevance, Oxford, Basil Blackwell.

HARRIS, B. and B. SHERWOOD (1978): «Translation as an Innate Skill», D. Gerver and H. W. Sinaiko (Eds.), Language Interpretation and Communication, New York, Plenum Press, pp. 155-170.

HATIM, B. and I. MASON (1990) : Discourse and the Translator, Londres, Longman.

HEWSON, L. and J. MARTIN (1991) : Redefining Translation. The Variational Approach, Londres, Routledge.

HOLMES, J. (1988) : «The Name and Nature of Translation Studies», J. Holmes, Translated! Papers in Literary Translation and Translation Studies, Amsterdam, Rodopi.

HOUSE, J. (1981) : A Model for Translation Quality Assessment, Tübingen, Gunter Narr.

JAKOBSON, R. (1959) : «On Linguistic Aspects of Translation», R. A. Brower (Ed.), On Translation, Harvard University Press, pp. 232-239.

KADE, O. (1968) : Zufall und Gesetzmässigkeit in der Übersetzung, Leipzig, Verlag Enzyklopädie.

KOLLER, W. (1979) : Einfïhrung in die Übersetzungswissenschaft, Heidelberg, Quelle \& Meyer.

LJDUSKANOV, A. (1969) : Traduction humaine et traduction mécanique, Centre de linguistique quantitative de la Faculté des Sciences de l'Université de Paris.

MAYORAL ASENSIO, R., KELLY, D. and N. GALLARDO (1988) : «Concept of Constrained Translation: Non-linguistic Perspectives of Translation», Meta, 33-3, pp. 356-367.

NEUBERT, A. (1968) : «Pragmatische Aspekte der Übersetzung», Fremdsprachen, 2, pp. 21-33.

NEUBERT, A. (1985) : Text and Translation, Leipzig, Verlag Enzyklopädie.

NEWMARK, P. (1988): A Texbook of Translation, Londres, Prentice Hall.

RABADAN, R. (1991) : Equivalencia y traducción, Universidad de León.

REISS, K. (1971) : Möglichkeiten und Grenzen der Übersetzungskritik, Munich, Hueber.

REISS, K. (1976) : Textyp und Übersetzungsmethode. Der operative Text, Kronberg, Scriptor.

REISS, K. (1981) : «Der Übersetzungsvergleich. Forme-Funktionen-Anwendbarkeit», W. Kühlwein, G. Thome, W. Wilss (Eds.), Kontrastive Linguistik und Übersetzungswissenschaft, Munich, Fink.

SNELL-HORNBY, M. (1988) : Translation Studies. An Integrated Approach, Amsterdam, John Benjamins.

STEINER, G. (1975) : After Babel, Oxford University Press.

TITFORD, CH. (1982) : «Sub-titling-Constrained Translation», Lebende Sprachen, 3, pp. 113-116.

WILSS, W. (1977) : Übersetzungswissenschaft. Probleme und Methoden, Stuttgart, Klett. 